\title{
Expression of Forkhead-box protein A1, a marker of luminal A type breast cancer, parallels low Oncotype DX 21-gene recurrence scores
}

\author{
Foluso O Ademuyiwa ${ }^{1}$, Mangesh A Thorat ${ }^{2}$, Rohit K Jain ${ }^{2}$, Harikrishna Nakshatri ${ }^{3,4}$ \\ and Sunil Badve ${ }^{2}$ \\ ${ }^{1}$ Department of Medicine, Indiana University, School of Medicine, Indianapolis, IN, USA; ${ }^{2}$ Department of \\ Pathology and Laboratory Medicine, Indiana University, School of Medicine, Indianapolis, IN, USA; \\ ${ }^{3}$ Department of Surgery, Indiana University School of Medicine, Indianapolis, IN, USA and ${ }^{4}$ Department of \\ Biochemistry and Molecular Biology, Indiana University School of Medicine, Indianapolis, IN, USA
}

\begin{abstract}
The Oncotype DX assay is one of the molecular tests that provide predictive and prognostic information to breast cancer patients with estrogen receptor (ER)-positive and node-negative disease. This study evaluates the association of Forkhead-box protein A1 (FOXA1) and GATA-binding protein 3 (GATA3) expressions with Oncotype DX recurrences scores in 77 cases of patients with ER-positive node-negative breast carcinomas diagnosed at Indiana University. The data were correlated with patient age, tumor size, histologic type, ScarffBloom-Richardson score, histologic grade, and progesterone receptor status. The median FOXA1 and GATA3 scores were $\mathbf{2 4 0}$ and $\mathbf{2 0 0}$, respectively. The Oncotype DX recurrence scores were low in $\mathbf{5 7 \%}$, intermediate in $30 \%$, and high in $13 \%$ of cases. FOXA1 expression correlated negatively with Oncotype DX recurrence scores $(P=0.004)$, and histologic type $(P=0.0004)$. Oncotype DX recurrences score also correlated negatively with progesterone receptor $(P=0.035)$ with $100 \%$ of progesterone receptor-negative cases having high or intermediate Oncotype DX scores. FOXA1 and GATA3 expressions correlated positively $(P=0.014)$. The correlation between FOXA1 expression and Oncotype DX recurrence scores remained significant after adjusting for multiple comparisons and controlling for confounders such as histological type, grade, and progesterone receptor. A statistically significant correlation between the Oncotype DX recurrence scores and FOXA1 expression in our diverse cohort of ER-positive breast cancer patients was observed. We propose that this may represent a more cost-effective strategy to further risk stratify patients with good prognosis in whom chemotherapy may be omitted. To confirm these findings, further studies in a larger cohort of patients are warranted.
\end{abstract}

Modern Pathology (2010) 23, 270-275; doi:10.1038/modpathol.2009.172; published online 27 November 2009

Keywords: FOXA1 expression; GATA3 expression; Oncotype DX; luminal A type breast cancer marker

In this era of personalized medicine, there has been a proliferation of molecular tests in breast cancer to

Correspondence: Dr S Badve, MD, Department of Pathology and Laboratory Medicine, Indiana University School of Medicine, 350 West 11th Street, CPL 4050, Indianapolis, IN 46202, USA.

E-mail: sbadve@iupui.edu

This work has been previously presented at ECOG Annual Meeting, 2008, Young Investigator Symposium.

Received 4 June 2009; revised 7 August 2009; accepted 8 August 2009; published online 27 November 2009 predict response to systemic therapy and also to offer prognostic information to patients. Several multigene prognostic assays are either currently available or are in development. The Oncotype DX recurrence assay (Genomic Health, Redwood, CA, USA) is one of the major commercially available prognostic tools licensed for use in the United States in patients with node-negative estrogen receptor (ER)-positive breast cancer. ${ }^{1}$ It assists clinicians in predicting the likelihood of recurrence as well as predicts the benefit of adjuvant systemic chemotherapy 
given in conjunction with hormonal manipulation. ${ }^{2}$ It is a reverse transcription-polymerase chain reaction assay that examines the expression of 16 cancerrelated genes (Ki67, STK15, survivin, CCNB1, MyBL2, MMP11, CTSL2, GRB7, Her2/neu, GSTM1, CD68, BAG1, ER, progesterone receptor [PR], BCL2, and SCUBE2) and 5 reference genes from malignant breast specimens. The expression pattern of each of these genes is tabulated using an equation and a recurrence score is assigned to each case. Among these genes, higher expression levels of $E R, P R$, BCL2, SCUBE2, GSTM1, and BAG1 reduce the recurrence score (negative values) whereas higher expression levels of remaining genes increase the recurrence score. Although a direct relationship between molecular subtypes and recurrence score has not been investigated, indirect evidence suggests that patients with low recurrence score belong to luminal A subtype. ${ }^{3}$ Patients in the high recurrence score category benefit from adjuvant chemotherapy; however, those with a low recurrence score tend to have a lower likelihood of benefiting from cytotoxic chemotherapy and are a potential group in whom chemotherapy can be avoided. Other assays, either available or in development, include the 70 gene profile/MammaPrint, Pro-Ex Br, wound response gene signature, intrinsic subtypes, Mammostrat, 2 gene ratio, MapQuant DX, and the invasiveness gene signature. ${ }^{4-10}$ Most of these assays, including the Oncotype DX assay, are expensive and have not been prospectively validated. In addition, some of these assays require the use of fresh tissue for processing. It therefore becomes necessary to develop novel strategies for providing both prognostic and predictive information to patients in a more cost-effective and efficient manner using techniques that can be clinically reproducible and prospectively validated. One such strategy could be to develop immunohistochemical assays that can identify luminal A subtype/low recurrence score tumors in which cytotoxic chemotherapy can safely be avoided.

The transcription factor network composed of ER, Forkhead-box protein A1 (FOXA1), and GATAbinding protein 3 (GATA3) controls the gene expression pattern in luminal subtype A breast cancers. ${ }^{11-13}$ This class of breast cancers tends to be ER-positive, PR-positive, Her2/neu-negative, well differentiated; therefore, it may correspond to the majority of cases sent for Oncotype DX testing in clinical practice. FOXA1 is a forkhead family transcription factor that interacts with cis-regulatory regions in chromatin and enhances the interaction of $\mathrm{ER} \alpha$ with chromatin. ${ }^{14}$ It is required for optimum expression of approximately $50 \%$ of ER-regulated genes and estrogen-induced cell proliferation. ${ }^{14,15} \mathrm{In}$ mammary epithelial cells, FOXA1 expression is positively regulated by GATA $3,{ }^{12}$ which is a lineage-restricted transcription factor essential for the differentiation of mammary luminal progenitor cells. $^{12,16}$ Therefore, luminal epithelial cells that express ER and GATA3 are expected to express the highest levels of FOXA1 in the mammary gland. Indeed, FOXA1, ER, and GATA3 are each expressed in a specific subpopulation of normal luminal epithelial cells and are likely coexpressed. ${ }^{12,17,18}$

As FOXA1, GATA3, and ER coexist in a network regulating luminal subtype A breast carcinomas, we hypothesized that tumors expressing FOXA1 and/or GATA3 will have a low recurrence score. We therefore investigated the correlation of FOXA1 and GATA3 expressions with the recurrence score in breast cancers that were tested by Oncotype DX. To our knowledge, this is the first attempt to correlate the recurrence score with novel molecular markers.

\section{Materials and methods}

The study population comprised 77 consecutive cases of invasive breast cancers that had Oncotype DX testing performed on tumor blocks between 2005 and 2008 from the pathology department of the Indiana University and affiliated hospitals. Oncotype DX was performed on paraffin-embedded tumor samples at Genomic Health Laboratories as described earlier. ${ }^{1}$ All patients had ER-positive and node-negative invasive breast cancers. The slides were reviewed by a breast pathologist and graded according to the modified Nottingham grading criteria. The study was approved by the Indiana University School of Medicine Institutional Review Board.

Her2/neu, ER, and PR status were obtained from the pathology reports and verified from the Oncotype DX report. ER and PR immunohistochemical staining at our institutions is performed using 1D5 mouse monoclonal and clone PR636 (Dako, Carpenteria, CA, USA) antibodies for ER and PR, respectively. Her2/neu protein is analyzed using the Hercept test (Dako) using standard FDA-approved methods. A positive result was indicated by $3+$ immunoreactivity (overexpression) or fluorescence in situ hybridization amplification.

Expression of FOXA1 was analyzed using goat antihuman FOXA1 antibody (Santa Cruz Biotechnology, Santa Cruz, CA, USA) by IHC. After dewaxing and hydration, $4 \mathrm{~mm}$ sections from formalinfixed paraffin embedded tissue were treated with target retrieval (Dako, pH 6.0), in a pressure cooker. Endogenous peroxidase activity was blocked by hydrogen peroxide for $10 \mathrm{~min}$. The slides were then incubated with polyclonal goat FOXA1 antibody (1:200; Santa Cruz Biotechnology) for $1 \mathrm{~h}$ at room temperature. The sections were incubated with donkey antigoat horseradish peroxidase polymer conjugate (Jackson Labs, West Grove, PA, USA) according to the manufacturer's instructions. The stain was developed using diaminobenzidine (DAB) plus (Dako, Glostrup, Denmark) and hematoxylin QS (Vector Laboratories, Burlingame, CA, USA) counterstain. To verify the specificity of staining, 
nonimmune goat serum and PBS-negative controls were used. Similar procedure was used for GATA3 using goat antihuman GATA3 antibody (1:100; Santa Cruz Biotechnology). Percentage of staining was categorized as ' $0 \%$ ' if there was no nuclear expression to a maximum score of ' $100 \%$ '. Intensity was scored as $1+, 2+$, and $3+$ for weak, moderate, and strong staining, respectively.

Percentage $(P)$ and intensity $(I)$ of nuclear expression for FOXA1 and GATA3 were multiplied to generate a numerical expression score $(S=P \times I)$. The maximum expression was therefore 300 , whereas the minimum was 0 . A cutoff of 30 was used for categorizing FOXA1 as positive. ${ }^{17}$ GATA3 was analyzed using a continuous variable. Oncotype DX results were obtained from the pathology files. The recurrence scores were used as a continuous variable as well as categorized as low (0-17), intermediate (18-30), or high ( $>30$ ).

Bivariate correlations of Oncotype DX recurrence scores, FOXA1, and GATA3 expressions with each other and with tumor type, grade, size, and PR were tested. Partial correlations of Oncotype DX recurrence scores, FOXA1, and GATA3 with each other were also tested controlling for variables correlating significantly with the variable in the pair being analyzed. $\chi^{2}$, ANOVA, and Kruskal-Wallis tests were appropriately applied for analysis of variables in Oncotype DX recurrence score categories. All tests were two sided and have been adjusted for multiple comparisons using Bonferroni-Holm's method. We used $5 \% \alpha$-level to determine significance. Data were analyzed using Statistical Package for Social Sciences 16.0 (SPSS Inc., Chicago, IL, USA).

\section{Results}

FOXA1 and GATA3 expressions were analyzed in 77 consecutive cases referred for Oncotype DX analysis between 2005 and 2008 by members of a multidisciplinary team specializing in the treatment of breast cancer at Indiana University and three other affiliated hospitals. Patient characteristics are shown in Table 1. Twenty-one of 77 cases $(27 \%)$ analyzed were $<50$ years of age; the median age was 55 years (mean, 55; range 29-77). Median tumor size was $1.4 \mathrm{~cm}$ (mean, $1.6 \mathrm{~cm}$; range, $0.3-7 \mathrm{~cm}$ ). Invasive ductal carcinomas accounted for $75 \%$ of tumors, whereas $9 \%$ were lobular, and $16 \%$ were mixed. Forty percent of tumors were histologic grade I, 46 and $14 \%$ were grades II and III, respectively. PR expression was absent in 11 (14\%) cases. Caucasians accounted for $82 \%$ of cases vs $5 \%$ African-Americans. Race was undocumented in $13 \%$.

Oncotype DX recurrence scores were low, intermediate, and high in 57,30 , and $13 \%$ of cases, respectively; the median recurrence score was 16 . Oncotype DX recurrence score categories did not differ by age or tumor type. However, there
Table 1 Patient characteristics

\begin{tabular}{lc}
\hline Age (mean, range) & $55(29-77)$ \\
$\quad<50$ & $21(27 \%)$ \\
$\geq 50$ & $56(73 \%)$ \\
& \\
Oncotype DX recurrence score (mean) & 18.8 \\
Low & $44(57 \%)$ \\
Intermediate & $23(30 \%)$ \\
High & $10(13 \%)$ \\
& \\
Histology & \\
Invasive ductal carcinoma & $58(75 \%)$ \\
Invasive lobular carcinoma & $7(9 \%)$ \\
Mixed & $12(16 \%)$ \\
Race & \\
Caucasian & \\
African-American & $63(82 \%)$ \\
Unknown & $4(5 \%)$ \\
Tumor size (mean, range) & $10(13 \%)$ \\
$\quad \leq 1 \mathrm{~cm}$ & \\
1.1-2 cm & $1.6 \mathrm{~cm}(0.3-7.0)$ \\
$>2$ cm & $20(26 \%)$ \\
PR & $39(51 \%)$ \\
Positive & $18(23 \%)$ \\
Negative & \\
Not assessable & \\
& \\
Grade & $64(83 \%)$ \\
I & $11(14 \%)$ \\
III & $2(3 \%)$ \\
& \\
&
\end{tabular}

was a significant difference across the categories when compared with tumor size and tumor grade including Scarff-Bloom-Richardson score. High Oncotype DX recurrence score category showed a higher median Scarff-Bloom-Richardson score. Oncotype DX recurrence score also correlated negatively with $\mathrm{PR}(P=0.035)$ with $100 \%$ of the PR-negative cases having a high or intermediate Oncotype DX score. Table 2 shows the relationship between the variables and Oncotype DX recurrence score categories.

The median FOXA1 score was 240 (range, 0-300). FOXA1 score negatively correlated with the recurrence score both using a categorical $(P<0.0001)$ and continuous variable $(P=0.004)$. A lower mean FOXA1 score was observed in high Oncotype DX recurrence score category (Figure 1a and b). Intermediate Oncotype DX recurrence score category had intermediate FOXA1 scores. Thus, FOXA1 score significantly differed among the three Oncotype DX recurrence scores categories. FOXA1 expression also correlated with histologic type $(P=0.0004)$; invasive ductal carcinoma (mean $=211.6)$ showing lower FOXA1 scores as compared to invasive lobular carcinoma (mean $=285.7)$ and mixed tumors showing intermediate FOXA1 scores $($ mean $=222)$.

The median GATA3 score was 200 with a similar range as FOXA1. GATA3 expression showed a 
negative trend with Oncotype DX recurrence score taken as a continuous variable (Figure 2a and b); however, this did not reach statistical significance $(P=0.236)$. Moreover, GATA3 expression did not significantly differ among the three Oncotype DX recurrence score categories $(P=0.204)$. GATA3 expression did not correlate with age, tumor size, histological type, grade, SBR score, and PR expression status. FOXA1 and GATA3 expressions were positively correlated $(P=0.014)$.

The correlation between FOXA1 expression and Oncotype DX recurrence score remained significant after adjusting for multiple comparisons. As either

Table 2 Oncotype DX recurrence score categories and frequencies of clinicopathologic variables

\begin{tabular}{lcccc}
\hline Variable & \multicolumn{4}{c}{ ODX RS categories } \\
\cline { 2 - 5 } & $\begin{array}{c}\text { Low } \\
(\mathrm{n}=44)\end{array}$ & $\begin{array}{c}\text { Intermediate } \\
(\mathrm{n}=23)\end{array}$ & $\begin{array}{c}\text { High } \\
(\mathrm{n}=10)\end{array}$ & P-value \\
\hline Age (mean) & 56 & 53 & 60 & $0.243^{\mathrm{a}}$ \\
Size (mean) & 1.5 & 1.4 & 2.8 & $<0.0001^{\mathrm{a}}$ \\
Grade (N, \%) & & & & \\
I & $22(50)$ & $9(39)$ & $0(0)$ & $0.026^{\mathrm{b}}$ \\
II & $19(43)$ & $9(39)$ & $7(70)$ & \\
III & $3(7)$ & $5(22)$ & $3(30)$ & \\
Histology (N, \%) & & & & \\
Ductal & $32(72)$ & $17(74)$ & $9(90)$ & $0.451^{\mathrm{b}}$ \\
Lobular & $6(14)$ & $1(4)$ & $0(0)$ & \\
Mixed & $6(14)$ & $5(22)$ & $1(10)$ & $0.050^{\mathrm{c}}$ \\
SBR score (median) & 1.5 & 2 & 2 & $0.0001^{\mathrm{a}}$ \\
FOXA1 expression & 235 & 228 & 135.5 & $<0.204^{\mathrm{a}}$ \\
GATA3 expression & 216.1 & 227 & 173.5 & \\
\hline
\end{tabular}

${ }^{\mathrm{a}}$ ANOVA.

${ }^{\mathrm{b}} \chi^{2}$ test.

${ }^{\mathrm{C}}$ Kruskal-Wallis test.
FOXA1 expression or Oncotype DX recurrence score correlated significantly with histological type, grade, and PR, we also tested correlation between FOXA1 and Oncotype DX recurrence score controlling for these variables individually and found that the correlation remained significant in all these analyses.

\section{Discussion}

Approximately, $50 \%$ of women with a new breast cancer diagnosis in the United States have ERpositive disease without regional lymph node metastasis. ${ }^{19}$ The current magnitude and public health burden of early-onset breast cancer underscore the necessity of further understanding the optimal management for this group of patients. Further recurrence risk reduction strategies before or after surgical treatment include the use of neoadjuvant or adjuvant therapy with hormonal manipulation or cytotoxic chemotherapy. Administration of tamoxifen in women with ER-positive disease reduces the annual breast cancer mortality by approximately $30 \%$ irrespective of the use of chemotherapy or age. ${ }^{20}$ The decision to administer adjuvant chemotherapy in this group of women by estimating the absolute benefit to be derived from such therapies becomes critically important given the significant improvements seen with hormonal therapies alone. In addition, chemotherapy is associated with both long- and short-term toxicities such as the risk of secondary malignancies, significant healthcare costs, and loss of productive time. Traditionally, adjuvant chemotherapy decisions are made using clinicopathological prognostic factors such as tumor size, grade, regional lymph node status, age, or the decision tools/guidelines based on these factors, e.g., Adjuvant! Online,
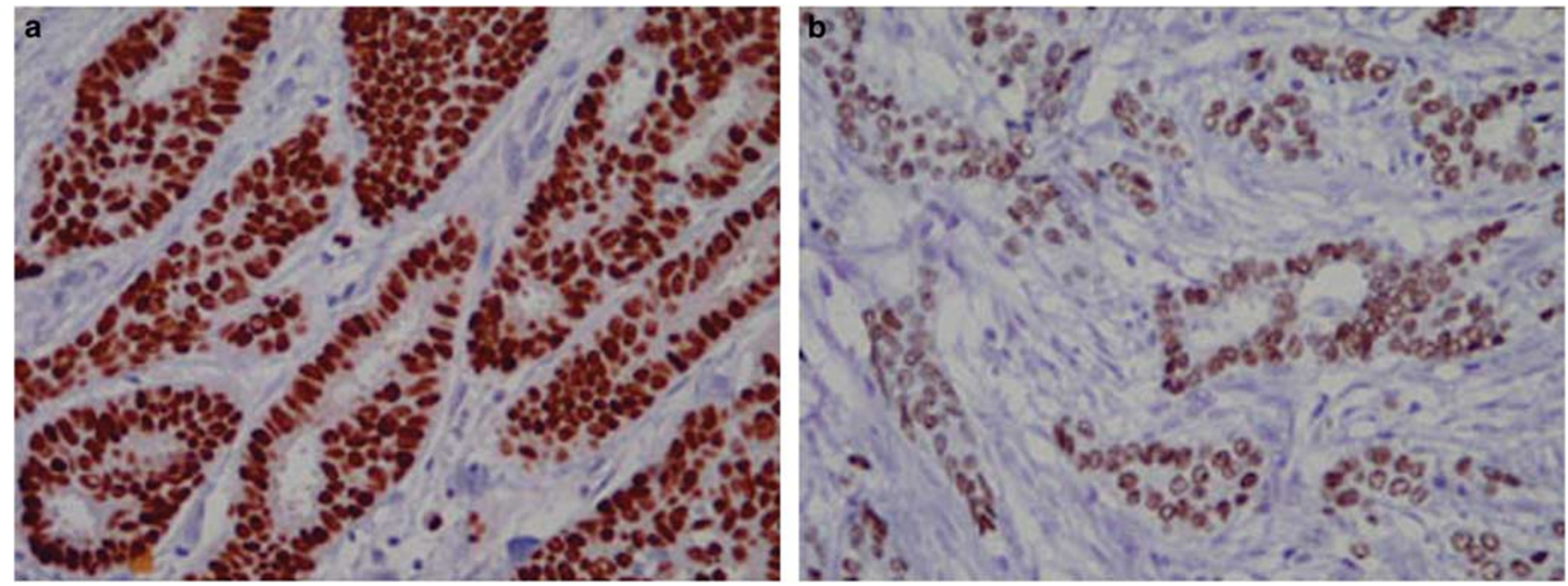

Figure 1 Immunohistochemical staining for FOXA1 (a) and GATA3 (b) in breast tumor from a case with low recurrence score (RS =10). Note the high level of expression of FOXA1 (score 300) and GATA3 (score 300) in the tumor cells. 

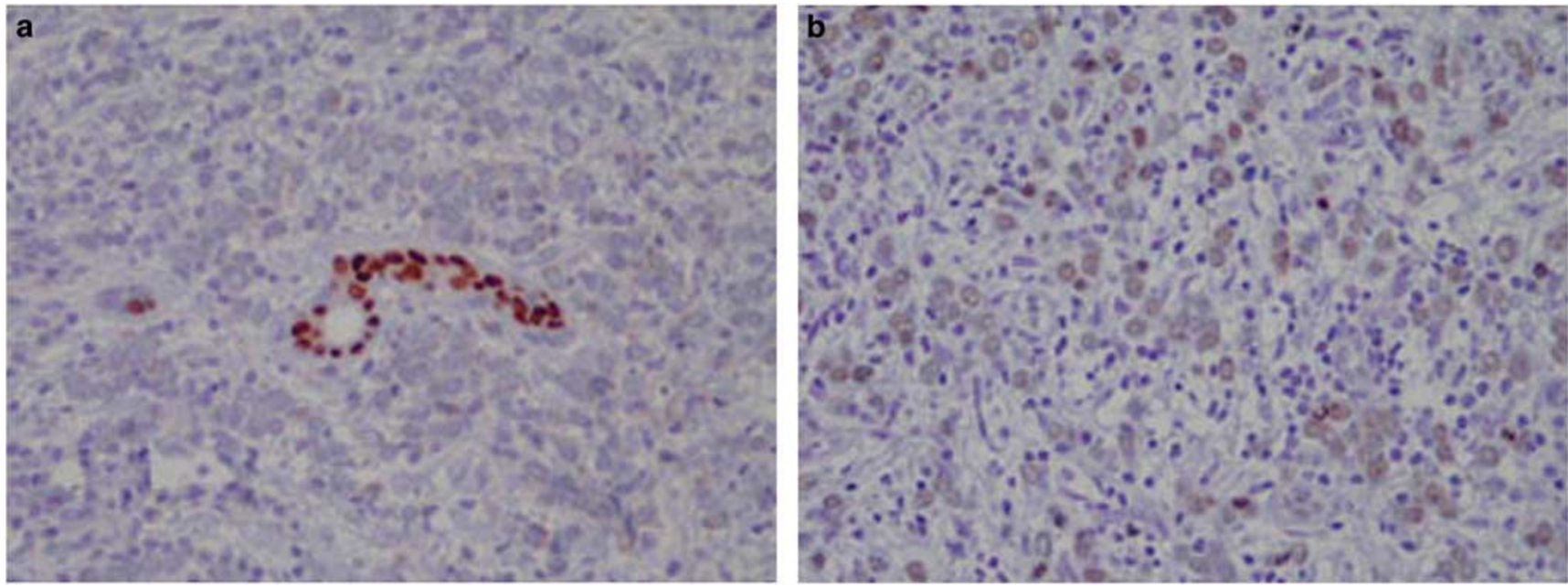

Figure 2 Immunohistochemical staining for FOXA1 (a) and GATA3 (b) in breast tumor from a case with high recurrence score (RS = 37). Note the lack of expression of FOXA1 and intermediate level expression of GATA3 in tumor cells. Strong nuclear expression of FOXA1 is, however, noted in the normal duct entrapped within the tumor.

National Comprehensive Cancer Network (NCCN), and St Gallen's guidelines. ${ }^{21-23}$

More recently, genetic profiling is being incorporated into clinical oncology practice to guide adjuvant therapy decisions. Oncotype DX recurrence score assay is one of the diagnostic tools recently approved in the United States for use in breast cancer patients with node-negative ER-positive tumors to guide adjuvant chemotherapy decisions. Although such tools may revolutionize the care of breast cancer patients by attempting to tailor therapy to each individual's molecular profile, they are expensive and currently lack prospective validation. Owing to these concerns, several investigators have attempted to use clinicopathologic features to predict the recurrence score. In a population-based cohort of 300 early-stage breast cancer patients, Wolf et $a 1^{24}$ determined that neither standard clinicopathologic features nor commonly used clinical guidelines reliably predicted the recurrence score. Another study by Flanagan et $\mathrm{l}^{25}$ suggested that the recurrence score correlated with histopathologic variables such as tubule formation, nuclear grade, mitotic count, ER status, PR status, and Her2/neu status. A hypothesis of the observed discrepancy may be that the molecular biology of these tumors may not correlate completely with standard histopathologic variables such as those that have been tested. Given that Oncotype DX evaluates prognosis by more formal molecular risk estimation in ER-positive breast cancers, ${ }^{1}$ FOXA1 and GATA3, which contribute to the hormonal control of gene expression pattern in this group of patients in conjunction with ER, may in fact correlate with the recurrence score.

In this study, we report on the correlation between FOXA1 and GATA3 expression levels and the Oncotype DX 21-gene recurrence scores in 77 consecutive breast cancer patients with ER-positive node-negative disease in a diverse population from different hospital settings. We did indeed find a statistically significant negative correlation between the recurrence scores and FOXA1 expression. GATA3 expression also correlated negatively with the recurrence scores; however, this did not reach statistical significance, possibly because of our limited sample size.

Another interesting observation of our study was that the proportion of patients with an intermediate recurrence score was higher than that reported in the analysis of the National Surgical Adjuvant Breast and Bowel Project (NSABP) B-14 clinical trial $(29.9 \%$ vs $22 \%){ }^{1}$ Similarly, the proportion of individuals with a high recurrence score was much lower in our cohort than in the patients on B-14 (13\% vs $27 \%)$. We also observed similar findings in the studies evaluating the influence of Oncotype DX recurrence score on decision making and its association with clinicopathologic features. ${ }^{24-27}$ Thus, our study likely represents a more 'real-life' distribution of Oncotype DX risk categories in patients with early-stage ER-positive disease.

We are not suggesting that patients with earlystage ER-positive breast cancer should currently be evaluated for FOXA1 and GATA3 expressions rather than the Oncotype DX multigene diagnostic assay; indeed our study does have certain limitations such as limited sample size, lack of prospective validation, and followup data. We do, however, suggest that if other cost-effective and reproducible techniques for providing prognostic information to patients and clinicians become available, then they ought to be investigated in greater depth for a possibility of replacing expensive molecular profiles. This becomes particularly critical in areas where Oncotype DX testing is not available or in individuals who may not have ready access to the test for a variety of reasons. 
To our knowledge, this is the first study correlating novel transcription factors and other clinicopathologic characteristics with the Oncotype DX recurrence scores. Although our data need to be reproduced in a larger cohort, this concept may indeed represent a novel alternate way to further stratify breast cancer patients to determine which patient might be at a lower risk of recurrence and therefore avoid chemotherapy in such patients.

\section{Disclosure/conflict of interest}

A patent application has been filed by Indiana University for the use of FOXA1 as a prognostic marker in breast cancer.

\section{References}

1 Paik S, Shak S, Tang G, et al. A multigene assay to predict recurrence of tamoxifen-treated, nodenegative breast cancer. $N$ Engl J Med 2004;351: 2817-2826.

2 Paik S, Tang G, Shak S, et al. Gene expression and benefit of chemotherapy in women with node-negative, estrogen receptor-positive breast cancer. J Clin Oncol 2006;24:3726-3734.

3 Fan C, Oh DS, Wessels L, et al. Concordance among gene-expression-based predictors for breast cancer. $\mathrm{N}$ Engl J Med 2006;355:560-569.

4 Ignatiadis M, Sotiriou C. Understanding the molecular basis of histologic grade. Pathobiology 2008;75: 104-111.

5 Loi S, Haibe-Kains B, Desmedt C, et al. Definition of clinically distinct molecular subtypes in estrogen receptor-positive breast carcinomas through genomic grade. J Clin Oncol 2007;25:1239-1246.

6 Ma XJ, Wang Z, Ryan PD, et al. A two-gene expression ratio predicts clinical outcome in breast cancer patients treated with tamoxifen. Cancer Cell 2004;5:607-616.

7 van de Vijver MJ, He YD, van't Veer LJ, et al. A geneexpression signature as a predictor of survival in breast cancer. N Engl J Med 2002;347:1999-2009.

8 Ross DT, Kim CY, Tang G, et al. Chemosensitivity and stratification by a five monoclonal antibody immunohistochemistry test in the NSABP B14 and B20 trials. Clin Cancer Res 2008;14:6602-6609.

9 Chang HY, Nuyten DS, Sneddon JB, et al. Robustness, scalability, and integration of a wound-response gene expression signature in predicting breast cancer survival. Proc Natl Acad Sci USA 2005;102:3738-3743.

10 Sorlie T, Perou CM, Tibshirani R, et al. Gene expression patterns of breast carcinomas distinguish tumor subclasses with clinical implications. Proc Natl Acad Sci USA 2001;98:10869-10874.

11 Eeckhoute J, Keeton EK, Lupien M, et al. Positive cross-regulatory loop ties GATA-3 to estrogen receptor alpha expression in breast cancer. Cancer Res 2007;67:6477-6483.

12 Kouros-Mehr H, Slorach EM, Sternlicht MD, et al. GATA-3 maintains the differentiation of the luminal cell fate in the mammary gland. Cell 2006;127: 1041-1055.

13 Thorat MA, Marchio C, Morimiya A, et al. Forkhead box A1 expression in breast cancer is associated with luminal subtype and good prognosis. J Clin Pathol 2008;61:327-332.

14 Carroll JS, Brown M. Estrogen receptor target gene: an evolving concept. Mol Endocrinol 2006;20:1707-1714.

15 Laganiere J, Deblois G, Lefebvre C, et al. From the Cover: location analysis of estrogen receptor alpha target promoters reveals that FOXA1 defines a domain of the estrogen response. Proc Natl Acad Sci USA 2005;102:11651-11656.

16 Asselin-Labat ML, Sutherland KD, Barker $\mathrm{H}$, et al. Gata-3 is an essential regulator of mammary-gland morphogenesis and luminal-cell differentiation. Nat Cell Biol 2007;9:201-209.

17 Badve S, Turbin D, Thorat MA, et al. FOXA1 expression in breast cancer-correlation with luminal subtype A and survival. Clin Cancer Res 2007;13:4415-4421.

18 Clarke RB, Howell A, Potten CS, et al. Dissociation between steroid receptor expression and cell proliferation in the human breast. Cancer Res 1997;57:4987-4991.

19 Jemal A, Siegel R, Ward E, et al. Cancer statistics, 2006. CA Cancer J Clin 2006;56:106-130.

20 Early Breast Cancer Trialists' Collaborative Group (EBCTCG). Effects of chemotherapy and hormonal therapy for early breast cancer on recurrence and 15year survival: an overview of the randomised trials. Lancet 2005;365:1687-1717.

21 Carlson RW, McCormick B. Update: NCCN breast cancer Clinical Practice Guidelines. J Natl Compr Cancer Netw 2005;3:S7-S11.

22 Goldhirsch A, Glick JH, Gelber RD, et al. Meeting highlights: international expert consensus on the primary therapy of early breast cancer 2005. Ann Oncol 2005;16:1569-1583.

23 Ravdin PM, Siminoff LA, Davis GJ, et al. Computer program to assist in making decisions about adjuvant therapy for women with early breast cancer. J Clin Oncol 2001;19:980-991.

24 Wolf I, Ben-Baruch N, Shapira-Frommer R, et al. Association between standard clinical and pathologic characteristics and the 21-gene recurrence score in breast cancer patients: a population-based study. Cancer 2008;112:731-736.

25 Flanagan MB, Dabbs DJ, Brufsky AM, et al. Histopathologic variables predict Oncotype DX recurrence score. Mod Pathol 2008;21:1255-1261.

26 Henry LR, Stojadinovic A, Swain SM, et al. The influence of a Gene expression profile on breast cancer decisions. J Surg Oncol 2009;99:319-323.

27 Oratz R, Paul D, Cohn AL, et al. Impact of a commercial reference laboratory test recurrence score on decision making in early-stage breast cancer. J Oncol Pract 2007;3:182-188. 